\title{
Causality and the Entropy-Complexity Plane: Robustness and Missing Ordinal Patterns
}

\author{
Osvaldo A. Rosso a,b,g,*, Laura C. Carpi ${ }^{\mathrm{c}, \mathrm{a}}$, Patricia M. Saco ${ }^{\mathrm{c}}$, \\ Martín Gómez Ravetti ${ }^{\mathrm{d}}$, Angelo Plastino ${ }^{\mathrm{e}, \mathrm{g}}$ and \\ Hilda A. Larrondo ${ }^{\mathrm{f}, \mathrm{g}}$ \\ a Departamento de Física, Instituto de Ciências Exatas. \\ Universidade Federal de Minas Gerais, \\ Av. Antônio Carlos, 6627 - Campus Pampulha. \\ 31270-901 Belo Horizonte - MG, Brazil. \\ ${ }^{\mathrm{b}}$ Chaos 8 Biology Group, Instituto de Cálculo, \\ Facultad de Ciencias Exactas y Naturales. \\ Universidad de Buenos Aires. \\ Pabellón II, Ciudad Universitaria. \\ 1428 Ciudad Autónoma de Buenos Aires, Argentina.

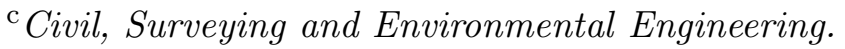 \\ The University of Newcastle. \\ University Drive, Callaghan NSW 2308, Australia. \\ ${ }^{\mathrm{d}}$ Departamento de Engenharia de Produção, \\ Universidade Federal de Minas Gerais, \\ Av. Antônio Carlos, 6627, Belo Horizonte, \\ 31270-901 Belo Horizonte - MG, Brazil. \\ ${ }^{\mathrm{e}}$ Instituto de Física, IFLP-CCT \\ Universidad Nacional de La Plata (UNLP). \\ C.C. 727, 1900 La Plata, Argentina. \\ ${ }^{\mathrm{f}}$ Facultad de Ingeniería, Universidad Nacional de Mar del Plata. \\ Av. J.B. Justo 4302, 7600 Mar del Plata, Argentina \\ ${ }^{\mathrm{g}}$ Fellow of CONICET-Argentina
}

\begin{abstract}
We deal here with the issue of determinism versus randomness in time series. One wishes to identify their relative weights in a given time series. Two different tools have been advanced in the literature to such effect, namely, i) the "causal" entropycomplexity plane [Rosso et al. Phys. Rev. Lett. 99 (2007) 154102] and ii) the estimation of the decay rate of missing ordinal patterns [Amigó et al. Europhys. Lett. 79 (2007) 50001, and Carpi et al. Physica A 389 (2010) 2020-2029]. In this work we extend the use of these techniques to address the analysis of deterministic finite time series contaminated with additive noises of different degree of correlation. The
\end{abstract}


chaotic series studied here was via the logistic map $(r=4)$ to which we added correlated noise (colored noise with $f^{-k}$ Power Spectrum, $0 \leq k \leq 2$ ) of varying amplitudes. In such a fashion important insights pertaining to the deterministic component of the original time series can be gained. We find that in the entropycomplexity plane this goal can be achieved without additional computations.

PACS: 05.45.Tp; 02.50.-r; 05.40.-a; 05.40.Ca;

\section{Introduction}

The concept of low dimensional deterministic chaos, derived from the modern theory of nonlinear dynamical systems, has changed our way of understanding and analyzing observational data $\mathcal{S}(t)$ (time series), leading to a paradigmchange from linear to non-linear approaches. Linear methods interpret observational signals from an underlying dynamical system that is regarded as being governed by a linear regime under which small perturbations lead to small effects. Consequently, all irregular behavior must be attributed to random external inputs [1]. However, chaos theory has shown that random inputs are not the only possible source of irregularities in a system's outputs. As a matter of fact, nonlinear deterministic autonomous equations of motion representing chaotic systems can give origin to very irregular signals. Of course, a system which has both nonlinear characteristics and random inputs will most likely produce irregular signals as well [1].

Chaotic time series are representative of a set of signals exhibiting complex non-periodic traces with continuous, broad band Fourier-spectra and displaying exponential sensitivity to small changes in the initial conditions. Clearly, signals emerging from chaotic time series occupy a place intermediate between (a) predictable regular or quasi-periodic signals and (b) totally irregular stochastic signals (noise) which are completely unpredictable. Chaotic time series are irregular in time, barely predictable, and exhibit interesting structures in the phase space.

Chaotic systems display "sensitivity to initial conditions" which manifests instability everywhere in the phase space and leads to non-periodic motion (chaotic time series). They display long-term unpredictability despite the

\footnotetext{
* Corresponding author

Email addresses: oarosso@fibertel.com.ar (Osvaldo A. Rosso), lauracarpi@gmail.com (Laura C. Carpi), Patricia.Saco@newcastle.edu.au (Patricia M. Saco), martin.ravetti@dep.ufmg.br (Martín Gómez Ravetti), plastino@fisica.unlp.edu.ar (Angelo Plastino), larrondo@fi.mdp.edu.ar (Hilda A. Larrondo).
} 
deterministic character of the temporal trajectory. In a system undergoing chaotic motion, two neighboring points in the phase space move away exponentially rapidly. Let $\mathbf{x}_{1}(t)$ and $\mathbf{x}_{2}(t)$ be two such points, located within a ball of radius $R$ at time $t$. Further, assume that these two points cannot be resolved within the ball due to poor instrumental resolution. At some later time $t^{\prime}$ the distance between the points will typically grow to $\left|\mathbf{x}_{1}\left(t^{\prime}\right)-\mathbf{x}_{2}\left(t^{\prime}\right)\right| \approx$ $\left|\mathbf{x}_{1}(t)-\mathbf{x}_{2}(t)\right| \exp \left(\lambda\left|t^{\prime}-t\right|\right)$, with $\lambda>0$ for a chaotic dynamics and $\lambda$ the biggest Lyapunov exponent. When this distance at time $t^{\prime}$ exceeds $R$, the points become experimentally distinguishable. This implies that instability reveals some information about the phase space population that was not available at earlier times [2].

The above considerations allow one to think of chaos as an information source. Moreover, the associated rate of generated information can be formulated in a precise way in terms of Kolmogorov-Sinai's entropy [34]. The KolmogorovSinai entropy measures the average loss of information rate. Its range of values goes from zero for regular dynamics, it is a positive number for chaotic system and, infinite for stochastic process. In consequence, if a dynamical system exhibits at least one positive Lyapunov exponent and a finite positive Kolmogorov-Sinai entropy one can assert that the system is deterministicchaotic.

Complex time series are very frequent in nature and also in man-made systems. The immediate question that emerges in relation to the underlying dynamical system that gives origin to these time series reads: Is the system chaotic (low-dimensional deterministic) or stochastic? Answering this question is important for a proper physical description of irregular dynamics. If one is able to show that the system is dominated by low-dimensional deterministic chaos, then only few (nonlinear and collective) modes are required to describe the pertinent dynamics [5]. If not, then the complex behavior could be modelled by a system dominated by a very large number of excited modes which are in general better described by stochastic or statistical approaches.

Even if several methodologies for evaluation of Lyapunov exponents and Kolmogorov-Sinai entropies for time-series' analysis have been proposed (see i.e [1]) their applicability involves taking into account constraints (stationarity, time series length, parameters values election for the methodology, etc.) which in general make the ensuing results non-conclusive. Thus, new tools for distinguishing chaos (determinism) from noise (stochastic) are needed.

In the early days of chaotic dynamics it was believed that obtaining finite, non-integer values for the fractal dimension was a strong indication of the presence of deterministic chaos, in opposition to systems whose dynamics are governed by stochastic process, thought to display an infinite value for the fractal dimension. However, Osborne and Provenzale [5] were able to present, 
in a seminal paper, a counter-example of the view that for a stochastic process one always detects a non-convergence of the correlation dimension (as the estimation of fractal dimension) in computed or measured time series. These researchers show that curves (time series) generated by inverting power-low spectra and random phases (colored random noises) are random fractal paths. Their Housdorf dimension is finite, and thus their correlation dimension is finite as well [5]. The main reason is that the Grassberger and Procaccia approach for the evaluation of the correlation dimension [6] is independent of the points-ordering in the signal and thus not able to test the differentiability of the curve under study. Thus, the Grassberger and Procaccia method cannot distinguish between fractal attractors and fractal curves if the two have the same dimension [5].

Among the many different tools advanced so as to distinguishing chaotic from stochastic time series we can mention:

(a) The proposal of Sugihara and May [7] based on nonlinear forecasting. The main idea is to compare predicted and actual trajectories. In this way, one can make tentative distinctions between dynamical chaos and measurement errors. For a chaotic time series the accuracy of nonlinear forecast diminishes for increasing prediction time-intervals (at a rate which yields an estimate of the Lyapunov exponent), whereas for uncorrelated noise, the forecasting accuracy does not exhibit such dependency [7].

(b) Kaplan and Glass [8,9] introduced a test based on the observation that the tangent to the trajectory generated by a deterministic system is a function of the position in phase space, and therefore, all the tangents to a trajectory in a given phase space region will display similar orientations, something that is not observed in stochastic dynamics.

Note that the two previously mentioned methods have in common the fact that one has to choose a certain length-scale $\epsilon$ and a particular embedding dimension $D$.

(c) More recently, Kantz and co-workers [1011] proposed to classify the signal behavior, without referring to any specific model, as stochastic or deterministic on a certain scale of resolution $\epsilon$, according to the dependence of the $(\epsilon, \tau)$ entropy $h(\epsilon, \tau)$ and finite-size Lyapunov exponent $\lambda(\epsilon)$ on $\epsilon$. Their methodology for distinguishing between chaos and noise is a refinement and generalization of the Grassberger and Procaccia one [6] for estimating the correlation dimension, and regarding finite values as signatures of deterministic behavior.

(d) The use of quantifiers based in Information Theory which incorporate in their evaluation the "time causality". We must note, that in this methodology a length scale $\epsilon$ is chosen (that means the scale at which one measure the time series), and also a particular embedding dimension $D$, however, 
its interpretation and main roll is completely different than in the previous enumerates methodologies (see for details see below).

The above mentioned quantifiers based on Information Theory concepts, are (1) "entropy", (2) "statistical complexity" and (3) the "entropy-complexity plane". Judicious use of this plane could i) lead to interesting insights into the characteristics of nonlinear chaotic dynamics, and ii) potentially improve our understanding of their associated time series. Moreover, these quantifiers can be used to detect determinism in time series [12]. In this vein we mention that Rosso et al. [12] found that different Information Theory based measures (normalized Shannon entropy and statistical complexity) allow for a better distinction between deterministic chaotic and stochastic dynamics when the so-called "causal" information is incorporated via Bandt and Pompe's (BP) methodology [13]. Indeed, new insight into the characterization of theoretical and observational time series, based on the Band and Pompe's methodology, reveals the emergence of "forbidden/missing patterns" [14,15,16,17]. The BP approach has been used also to distinguish deterministic behavior (chaos) from randomness in finite time series contaminated with observational white noise (uncorrelated noise) [14,15,18] by recourse to the analysis of the decay rate of the "missing ordinal patterns" as a function of the time series length.

Additionally, Zanin [19] and Zunino et al. [20] have recently studied the appearance of missing ordinal patterns in financial time series. They found evidence for the existence of deterministic forces in the medium- and long term dynamics. Moreover, they propose that an analysis of the number of missing patterns' evolution should be a useful tool in order to quantify the randomness of certain time-periods within a financial series. Also, the presence of missing ordinal patterns has been recently construed as possible evidence of deterministic dynamics in epileptic states. It is suggested in [21] that a missing patterns' quantifier could be regarded as a predictor of epileptic absence-seizures. It is of the essence to point out that in such researches only non correlated noise (white noise) has been considered, which makes the associated results somewhat incomplete, since the presence of colored noise has not been taken into account.

It becomes necessary then, and such is the objective of this work, to investigate from such viewpoint the robustness of the entropy-complexity causality plane, that plays a prominent role in some of the above cited discoveries. We intend to do this by analyzing the planes's ability to distinguish between noiseless chaotic time series and the ones that are contaminated with additive correlated noise (noise with power low spectrum $f^{-k}$ ).

The chaotic series studied here were generated by recourse to a logistic map to which noise with varying amplitudes was added. The decay rate of missing ordinal patterns was also investigated as a tool to distinguish among these 
systems.

The present paper is organized as follows: Section 2 gives a brief description of the Information Theory quantifiers used in this work. Section 3 presents the Bandt and Pompe methodology used for the evaluation of the quantifiers. The methodological framework used in this study is delineated in Section 4. Finally Sections 5 and 6 present the discussion of the results and the conclusions, respectively.

\section{Information Theory based quantifiers}

\subsection{Entropy and Statistical Complexity}

The information content of a system is typically evaluated via a probability distribution function (PDF) describing the apportionment of some measurable or observable quantity. An information measure can primarily be viewed as a quantity that characterizes this given probability distribution $P$. The Shannon entropy is very often used as a the "natural" one [22]. Given any arbitrary discrete probability distribution $P=\left\{p_{i}: i=1, \cdots, M\right\}$, with $M$ the number of freedom-degrees, Shannons logarithmic information measure reads

$$
\mathrm{S}[P]=-\sum_{i=1}^{M} p_{i} \ln \left(p_{i}\right) .
$$

It can be regarded as a measure of the uncertainty associated to the physical process described by $P$. From now on we assume that the only restriction on the PDF representing the state of our system is $\sum_{j=1}^{N} p_{j}=1$ (micro-canonical representation). If $\mathrm{S}[P]=\mathrm{S}_{\min }=0$ we are in position to predict with complete certainty which of the possible outcomes $i$, whose probabilities are given by $p_{i}$, will actually take place. Our knowledge of the underlying process described by the probability distribution is maximal in this instance. In contrast, our knowledge is minimal for a uniform distribution and the uncertainty is maximal, $\mathrm{S}\left[P_{e}\right]=\mathrm{S}_{\max }$.

It is widely known that an entropic measure does not quantify the degree of structure or patterns present in a process [23]. Moreover, it was recently shown that measures of statistical or structural complexity are necessary for a better understanding of chaotic time series because they are able capture their organizational properties [24]. This specific kind of information is not revealed by randomness' measures. The opposite extreme perfect order (like a periodic sequence) and maximal randomness (fair coin toss) possess no complex structure and exhibit zero statistical complexity. States between these 
extremes, a wide range of possible degrees of physical structure exists, that should be quantified by the statistical complexity measure. Rosso and coworkers introduced an effective statistical complexity measure (SCM) that is able to detect essential details of the dynamics and differentiate different degrees of periodicity and chaos [25]. This specific SCM, abbreviated as the MPR one, provides important additional information regarding the peculiarities of the underlying probability distribution, not already detected by the entropy.

The MPR-statistical complexity measure is defined, following the seminal, intuitive notion advanced by López-Ruiz et al. [26], via the product

$$
\mathcal{C}_{J S}[P]=\mathcal{Q}_{J}\left[P, P_{e}\right] \cdot \mathcal{H}_{S}[P]
$$

of i) the normalized Shannon entropy

$$
\mathcal{H}_{S}[P]=\mathrm{S}[P] / \mathrm{S}_{\max }
$$

with $\mathrm{S}_{\max }=\mathrm{S}\left[P_{e}\right]=\ln M,\left(0 \leq \mathcal{H}_{S} \leq 1\right)$ and $P_{e}=\{1 / M, \cdots, 1 / M\}$ (the uniform distribution) and ii) the so-called disequilibrium $\mathcal{Q}_{J}$. This quantifier is defined in terms of the extensive (in the thermodynamical sense) JensenShannon divergence $\mathcal{J}\left[P, P_{e}\right]$ that links two PDFs. We have

$$
\mathcal{Q}_{J}\left[P, P_{e}\right]=Q_{0} \cdot \mathcal{J}\left[P, P_{e}\right]
$$

with

$$
\mathcal{J}\left[P, P_{e}\right]=\mathrm{S}\left[\left(P+P_{e}\right) / 2\right]-\mathrm{S}[P] / 2-\mathrm{S}\left[P_{e}\right] / 2 .
$$

$Q_{0}$ is a normalization constant, equal to the inverse of the maximum possible value of $\mathcal{J}\left[P, P_{e}\right]$. This value is obtained when one of the values of $P$, say $p_{m}$, is equal to one and the remaining $p_{i}$ values are equal to zero, i.e.,

$$
Q_{0}=-2\left\{\left(\frac{M+1}{M}\right) \ln (M+1)-2 \ln (2 M)+\ln M\right\}^{-1} .
$$

The Jensen-Shannon divergence, that quantifies the difference between two (or more) probability distributions, is especially useful to compare the symbolcomposition of different sequences [27]. The complexity measure constructed in this way has the intensive property found in many thermodynamic quantities [25]. We stress the fact that the statistical complexity defined above is the product of two normalized entropies (the Shannon entropy and JensenShannon divergence), but it is a nontrivial function of the entropy because it depends on two different probabilities distributions, i.e., the one corresponding to the state of the system, $P$, and the uniform distribution, $P_{e}$. 


\subsection{Entropy-Complexity plane}

In statistical mechanics one is often interested in isolated systems characterized by an initial, arbitrary, and discrete probability distribution. Evolution towards equilibrium is to be described, as the overriding goal. At equilibrium, we can think, without loose of generality, that this state is given by the uniform distribution $P_{e}$. The temporal evolution of the statistical complexity measure $(\mathrm{SCM})$ can be analyzed using a diagram of $\mathcal{C}_{J S}$ versus time $t$. However, it is well known that the second law of thermodynamics states that for isolated systems entropy grows monotonically with time $\left(d \mathcal{H}_{S} / d t \geq 0\right)$ [28]. This implies that $\mathcal{H}_{S}$ can be regarded as an arrow of time, so that an equivalent way to study the temporal evolution of the SCM is through the analysis of $\mathcal{C}_{J S}$ versus $\mathcal{H}_{S}$. In this way, the normalized entropy-axis substitutes for the timeaxis. Furthermore, it has been shown that for a given value of $\mathcal{H}_{S}$, the range of possible statistical complexity values varies between a minimum $\mathcal{C}_{\text {min }}$ and a maximum $\mathcal{C}_{\text {max }}$ [29], restricting the possible values of SCM in this plane.

Therefore, the evaluation of the complexity provides additional insight into the details of the systems probability distribution, which is not discriminated by randomness measures like the entropy [12,24]. It can also help to uncover information related to the correlational structure between the components of the physical process under study [30]. The entropy-complexity diagram (or plane), $\mathcal{H}_{S} \times \mathcal{C}_{J S}$, has been used to study changes in the dynamics of a system originated by modifications of some characteristic parameters (see for instance Refs. [29,31,32,33] and references therein).

\subsection{Estimation of the Probability Distribution Function}

In using quantifiers based on Information Theory (like $\mathcal{H}_{S}$ and $\mathcal{C}_{J S}$ ), a probability distribution associated to the time series under analysis should be provided beforehand. The determination of the most adequate PDF is a fundamental problem because $P$ and the sample space $\Omega$ are inextricably linked. Many methods have been proposed for a proper selection of the probability space $(\Omega, P)$. We can mention: (a) frequency counting [34], (b) procedures based on amplitude statistics [35], (c) binary symbolic dynamics [36], (d) Fourier analysis [37] and, (e) wavelet transform [38], among others. Their applicability depends on particular characteristics of the data, such as stationarity, time series length, variation of the parameters, level of noise contamination, etc. In all these cases the dynamics' global aspects can be somehow captured, but the different approaches are not equivalent in their ability to discern all the relevant physical details. One must also acknowledge the fact that the above techniques are introduced in a rather "ad hoc fashion" and 
they are not directly derived from the dynamical properties themselves of the system under study.

Methods for symbolic analysis of time series that discretize the raw series and transform it into a sequence of symbols constitute a powerful tool. They efficiently analyze nonlinear data and exhibit low sensitivity to noise [39. However, finding a meaningful symbolic representation of the original series is not an easy task [40,41]. To our best knowledge, the Bandt and Pompe approach is the only symbolization technique, among those in popular use, that takes into account time-causality in the evaluation of the PDF associated to the time series of a given system [13. The symbolic data is i) created by associating a rank to the series-values and ii) defined by reordering the embedded data in ascending order. Data are reconstructed with an embedding dimension $D$ (see definition and methodological details below). In this way it is possible to quantify the diversity of the ordering symbols (patterns) derived from a scalar time series, evaluating the so called permutation entropy $\mathcal{H}_{s}$, and permutation statistical complexity $\mathcal{C}_{J S}$ (i.e., the normalized Shannon entropy and MPR-statistical complexity evaluated for the Bandt and Pompe's PDF).

\section{The Bandt and Pompe methodology}

\subsection{PDF based on ordinal patterns}

To use the Bandt and Pompe [13] methodology for evaluating of the probability distribution $P$ associated to the time series (dynamical system) under study, one starts by considering partitions of the pertinent $D$-dimensional space that will hopefully "reveal" relevant details of the ordinal-structure of a given one-dimensional time series $\mathcal{S}=\left\{x_{t}: t=1, \cdots, N\right\}$ with embedding dimension $D>1$. We are interested in "ordinal patterns" of order $D$ [13,45] generated by

$$
(s) \mapsto\left(x_{s-(D-1)}, x_{s-(D-2)}, \cdots, x_{s-1}, x_{s}\right)
$$

which assigns to each time $s$ the $D$-dimensional vector of values at times $s, s-1, \cdots, s-(D-1)$. Clearly, the greater the $D$-value, the more information on the past is incorporated into our vectors. By "ordinal pattern" related to the time $(s)$ we mean the permutation $\pi=\left(r_{0}, r_{1}, \cdots, r_{D-1}\right)$ of $[0,1, \cdots, D-1]$ defined by

$$
x_{s-r_{D-1}} \leq x_{s-r_{D-2}} \leq \cdots \leq x_{s-r_{1}} \leq x_{s-r_{0}}
$$


In order to get a unique result we set $r_{i}<r_{i-1}$ if $x_{s-r_{i}}=x_{s-r_{i-1}}$. This is justified if the values of $x_{t}$ have a continuous distribution so that equal values are very unusual. Otherwise, it is possible to break these equalities by adding small random perturbations.

Thus, for all the $D$ ! possible permutations $\pi$ of order $D$, their associated relative frequencies can be naturally computed by the number of times this particular order sequence is found in the time series divided by the total number of sequences. The probability distribution $P=\{p(\pi)\}$ is defined by

$$
p(\pi)=\frac{\sharp\{s \mid s \leq M-D+1 ;(s), \text { has type } \pi\}}{M-D+1} .
$$

In this expression, the symbol $\sharp$ stands for "number".

The procedure can be better illustrated with a simple example; let us assume that we start with the time series $\{1,3,5,4,2,5, \ldots\}$, and we set the embedding dimension $D=4$. In this case the state space is divided into 4! partitions and 24 mutually exclusive permutation symbols are considered. The first 4-dimensional vector is $(1,3,5,4)$. According to Eq. (7) this vector corresponds with $\left(x_{s-3}, x_{s-2}, x_{s-1}, x_{s}\right)$. Following Eq. (8) we find that $x_{s-3} \leq x_{s-2} \leq x_{s} \leq x_{s-1}$. Then, the ordinal pattern which allows us to fulfill Eq. (8) will be $[3,2,0,1]$. The second 4 -dimensional vector is $(3,5,4,2)$, and $[0,3,1,2]$ will be its associated permutation, and so on. For the computation of the Bandt and Pompe PDF we follow the very fast algorithm described by Keller and Sinn [45], in which the different ordinal patterns are generated in lexicographic ordering.

The Bandt and Pompe's methodology is not restricted to time series representative of low dimensional dynamical systems but can be applied to any type of time series (regular, chaotic, noisy, or reality based), with a very weak stationary assumption (for $k=D$, the probability for $x_{t}<x_{t+k}$ should not depend on $t$ [13]). It also assumes that enough data are available for a correct embedding procedure. Of course, the embedding dimension $D$ plays an important role in the evaluation of the appropriate probability distribution because $D$ determines the number of accessible states $D$ !. It also conditions the minimum acceptable length $M \gg D$ ! of the time series that one needs in order to work with reliable statistics.

The main difference between Information Theory quantifiers evaluated with the Bandt and Pompe-PDF and other PDFs (like a histogram-PDF), is that they are invariant with respect to strictly monotonous distortions of the data. This point is very important for the analysis of observed natural time series (like for example, sedimentary data related to rainfall by an unknown nonlinear function [46]). However, due to its invariance properties, quantifiers based on 
the Bandt and Pompe's approach, when applied to any other series that is strictly monotonic in the given data, will yield the same results. Therefore, in general, ordinal data processing tools based on ranked numbers lead to results that could be more useful for data analysis than those obtained using tools based on metric properties.

\subsection{Forbidden and Missing Ordinal Patterns}

As shown recently by Amigó et al. [14,15,16,17, in the case of deterministic one-dimensional maps, not all the possible ordinal patterns can be effectively materialized into orbits, which in a sense makes these patterns "forbidden". Indeed, the existence of these forbidden ordinal patterns becomes a persistent fact that can be regarded as a "new" dynamical property. Thus, for a fixed pattern-length (embedding dimension $D$ ) the number of forbidden patterns of a time series (unobserved patterns) is independent of the series length $N$. Remark that this independence does not characterize other properties of the series such as proximity and correlation, which die out with time [15]17. For example, in the time series generated by the logistic map $x_{k+1}=4 x_{k}\left(1-x_{k}\right)$, if we consider patterns of length $D=3$, the pattern $\{2,1,0\}$ is forbidden. That is, the pattern $x_{k+2}<x_{k+1}<x_{k}$ never appears [15].

Stochastic process could also present forbidden patterns [47]. However, in the case of either uncorrelated or correlated stochastic processes (white noise vs. noise with power low spectrum $f^{-k}$ with $k \geq 0$, ordinal Brownian motion, fractional Brownian motion, and fractional Gaussian noise) it can be numerically ascertained that no forbidden patterns emerge. Moreover, analytical expressions can be derived [48] for for some stochastic process (i.e. fractional Brownian motion for PDF's based on ordinal patterns with length $2 \leq D \leq 4$ ).

In the case of time series generated by unconstrained stochastic process (uncorrelated process) every ordinal pattern has the same probability of appearance [14,15, 16,17. That is, if the data set is long enough, all the ordinal patterns will eventually appear. In this case, when the number of time-series' observations becomes long enough the associated probability distribution function should be the uniform distribution, and the number of observed patterns will depend only on the length $N$ of the time series under study.

For correlated stochastic processes the probability of observing individual pattern depends not only on the time series length $N$ but also on the correlation structure [18]. The existence of a non-observed ordinal pattern does not qualify it as "forbidden", only as "missing", and it could be due to the finite length of the time series. A similar observation also holds for the case of real data that always possess a stochastic component due to the omnipresence of 
dynamical noise [42,43,44]. Thus, the existence of "missing ordinal patterns" could be either related to stochastic processes (correlated or uncorrelated) or to deterministic noisy processes, which is the case for observational time series.

Amigó and co-workers [14,15] proposed a test that uses missing ordinal patterns to distinguish determinism (chaos) from pure randomness in finite time series contaminated with observational white noise (uncorrelated noise). The test is based on two important practical properties: their finiteness and noise contamination. These two properties are important because finiteness produces missing patterns in a random sequence without constrains, whereas noise blurs the difference between deterministic and random time-series. The methodology proposed by Amigó et al. [15] consists in a graphic comparison between the decay of the missing ordinal patterns (of length $D$ ) of the time series under analysis as a function of the series length $N$, and the decay corresponding to white Gaussian noise. This methodology was recently extended by Carpi et al. [18 for the analysis of missing ordinal patterns in stochastic processes with different degrees of correlation: fractional Brownian motion (fBm), fractional Gaussian noise (fGn) and, noises with $f^{-k}$ power spectrum $(\mathrm{PS})$ and $(k \geq 0)$. Specifically, this paper analyzes the decay rate of missing ordinal patterns as a function of pattern-length $D$ (embedding dimension) and of time series length $N$. Results show that for a fixed pattern length, the decay of missing ordinal patters in stochastic processes depends not only on the series length but also on their correlation structures. In other words, missing ordinal patters are more persistent in the time series with higher correlation structures. Ref. [18] also has shown that the standard deviation of the estimated decay rate of missing ordinal patterns $(\alpha)$ decreases with increasing $D$. This is due to the fact that longer patterns contain more temporal information and are therefore more effective in capturing the dynamics of time series with correlation structures.

\section{Application to the logistic map with additive noise}

The logistic map constitutes a canonic example, often employed to illustrate new concepts and/or methods for the analysis of dynamical systems. Thus, we will use the logistic map with additive correlated noise in order to exemplify the behavior of the normalized Shannon entropy $\mathcal{H}_{S}$ and the Statistical Complexity $\mathcal{C}_{J S}$, both evaluated using a PDF based on the Bandt-Pompe's procedure. We will also investigate the decay rate of "missing ordinal patterns" in both a) the logistic map with additive noise and b) a pure-noise series.

The logistic map is a polynomial mapping of degree $2, F: x_{n} \rightarrow x_{n+1}$ [49], described by the ecologically motivated, dissipative system represented by the 
first-order difference equation

$$
x_{n+1}=r \cdot x_{n} \cdot\left(1-x_{n}\right)
$$

with $0 \leq x_{n} \leq 1$ and $0 \leq r \leq 4$

Let $\eta^{(k)}$ be a correlated noise with $f^{-k}$ power spectra generated as describe in, for instance, [12]. The steps to be followed are enumerated below.

(1) Using the Mersenne twister generator [50] through the MatLaB ${ }^{\complement}$ RAND function we generate pseudo random numbers $y_{i}^{0}$ in the interval $(-0.5,0.5)$ with an (a) almost flat power spectra (PS), (b) uniform PDF, and (c) zero mean value.

(2) Then, the Fast Fourier Transform (FFT) $y_{i}^{1}$ is first obtained and then multiplied by $f^{-k / 2}$, yielding $y_{i}^{2}$;

(3) Now, $y_{i}^{2}$ is symmetrized so as to obtain a real function. The pertinent inverse FFT is obtained, after discarding the small imaginary components produced by our numerical approximations.

(4) The resulting noisy time series is re-scaled to the interval $[-1,1]$, which produces a new time series $\eta^{(k)}$ that exhibits the desired power spectra and, by construction, is representative of non-Gaussian noises.

We considered a time series $\mathcal{S}=\left\{S_{n}, n=1, \cdots, N\right\}$ generated by the discrete system:

$$
S_{n}=x_{n}+A \cdot \eta_{n}^{(k)}
$$

in which, $x_{n}$ is given by the logistic map and $\eta_{n}^{(k)} \in[-1,1]$ represents a noise with power spectrum $f^{-k}$ and amplitude $A$.

In generating the logistic map's component of our time-series we fixed $r=4$ and started the iteration procedure with a random initial condition. The first 5 . $10^{4}$ iterations were considered part of the transient behavior and were therefore discarded. After the transient part died out, $N=10^{5}$ data were generated. In the case of the stochastic component of our time-series we considered $0 \leq k \leq$ 2 with $k$-values changing by the amount $\Delta k=0.25$. The noise amplitude was varied between $0 \leq A \leq 1$ with increments $\Delta A=0.1$. Ten noise time series of length $N=10^{5}$ data (different seeds) were generated for each value of the $k$-exponent. Figures 1 and 2 display the first return map corresponding to a typical signal obtained from Eq. (11) (we show only the first $N=10^{4}$ data points), for the cases of $k=0$ (white noise) and $k=1$ respectively. These graphs clearly show how the distorting effect of the additive noise on the logistic time series increases as the amplitude $A$ grows. 


\section{$5 \quad$ Results and discussion}

An important quantity for us, called $\mathcal{M}(N, D)$, is the average number of missing ordinal patterns of length $D$, that is, patterns which are not observed in a time series (TS) with $N$ data values. As we mentioned before, for pure correlated stochastic processes the probability of observing an individual pattern of length $D$ depends on the time series-length $N$ and on the correlation structure (as determined by the type of noise $k>0$ ). In fact, as shown for noises with an $f^{-k}$ Power Spectrum in Ref. [18, as the value of $k>0$ augments which implies that correlations grow - increasing values of $N$ are needed if one wishes to satisfy the "ideal" condition $\mathcal{M}(N, D)=0$. If the time series is chaotic but has an additive stochastic component, then one expects that as the time series' length $N$ increases, the number of "missing ordinal patterns" will decrease and eventually vanish. That this may happen does not only depend on the length $N$ but on the de correlation-structure of the added noise as well.

Here from we will fix the pattern-length at $D=6$ and the time series (TS) length at $N=10^{5}$ data. In Fig. 3 the average number of missing patterns

$<\mathcal{M}(N, D)>_{k=0}$ (mean value and standard deviation over ten time series (see Eq. (11)) is displayed as a function of the number of TS-data. $\mathcal{M}$ was evaluated as a function of the TS-length $10^{3} \leq N \leq 10^{5}$ with $\Delta N=20 . A=0$ corresponds to the case of the pure logistic time series and consequently the number of missing patterns is constant $\langle\mathcal{M}\rangle=645$ (see Fig. 3. a). From this plot we gather that the mean number of missing patterns rapidly decreases as the noise amplitude increases, and could vanish when the time series' length is large enough.

We pass now to consider the quantity $<\mathcal{M}(N, D)>_{k \neq 0}$ (see Eq. [11)] corresponding to the TS-number of missing patterns (mean value and standard deviation) as a function of the noise amplitude $A$. This is the purpose of Fig. 4. Due to the finiteness of the record length $N$ we find a non zero number of missing patterns. The noise correlation effect is more significant for values of $k>1$. This graph illustrates on the dependence of the number of missing patterns on $i$ ) the noise amplitude $A$ and $i$ ) the noise-correlation $k$.

Let us now focus our attention on how the average missing ordinal patterns $<\mathcal{M}(N, D)>$ decays as $N$ grows (for a fixed value of the pattern length $D$ ) by recourse to the exponential law advanced by Amigó et al. [15,16] and Carpi et al. [18], namely,

$$
\mathcal{M}(N, D)=B \cdot \exp \{-\alpha \cdot N\}
$$

where $\alpha \geq 0$ is the characteristic decay rate and $B$ is a constant. $\alpha$ is deter- 
mined by fitting Eq. (12) to the numerically generated values of $<\mathcal{M}(N, D)>$ (for each $k$ and $A$ ) using the least square method. Figure 5 displays the $\alpha$-values for different $k$-values as a function of the noise amplitude $A$. In the same plot we also represent, as horizontal lines, the corresponding values of the decay rate $\alpha$ pertaining to an strictly noisy TS. In all cases, for the evaluation of $\mathcal{M}(N, D)$ we consider $10^{3} \leq N \leq 10^{5}$, with $\Delta N=20$ and $D=6$. The mean value of the $r^{2}$ coefficient of the exponential fit (the "goodness" of the fit) was always larger than 0.95 .

We see in Fig. 5 that, if $A=0$ (logistic series with no noise), the decay rate vanishes. For $A \neq 0$ strictly forbidden patterns do not exist $(\alpha>0)$. Missing patterns are those that had not appeared before due to the finite TS-length' effect. When the amplitude of the added noise increases, the number of these missing patterns tends to decrease. However, their observation-probability depends on the time series' length $(N)$ and on the noise-correlation $(k)$.

We can regard the additive noise as a perturbation to the logistic data (see Figs. 1 and 2). As a result of this perturbation the original patterns can change and so does the PDF associated to the TS. The number of observed patterns becomes now a combination of those originated by, respectively, the deterministic and the stochastic series' components (their interaction may play a role as well). Note that the added noise can destroy true forbidden patterns, although it can create also new forbidden patterns. It is clear from Figs. 4 and 5 that, for a fixed number of data, the number of missing patterns tends to decrease and the corresponding decay rate $\alpha>0$, displays smaller values if both the noise amplitude $A$ and the correlation $k$ increase. However, as expected, for any given value of $k$, the logistic series contaminated with noise has a lower decay rate than that of the strictly noisy one. If the logistic map is contaminated with non correlated noise $(k=0)$ then, as Fig. 5 tells us, $\alpha<\alpha^{(k=0)}$ for all noise amplitudes $(0<A \leq 1)$ and deterministic influences on the TS can be established via the distance from the $\alpha$-decay curve to the strictly stochastic horizontal lines.

In a real situation, of course, the strength of the contaminating noise is unknown. However, were we are able to forecast an estimation or at least make an educated guess of the power factor $k$ of the contaminating noise, our results would motivate us to conjecture that:

- Given an observational time series of length $N$ and missing patterns decay rate $\alpha^{(T S)}$, and

- a pure $k$-noise time series of the same length $N$, with $\alpha^{(k-n o i s e)}$,

- if $\alpha^{(T S)}<\alpha^{(k-n o i s e)}$, then the observational time series can be associated to some deterministic process with an additive noise component.

Summing up, if one employs for the TS-analysis i) quantifiers based on In- 
formation Theory and ii) the Bandt and Pompe's PDF, several effects have to be considered, namely, those associated to the (a) finite number of data $N$; (b) noise contamination level (represented by $A$ ); (c) noise correlation (represented by $k$ ); and (d) persistence of forbidden patterns linked to the deterministic component of the series.

The missing patterns' quantifier $\alpha$ can be used for practical proposes in order to characterize an observational time series. However, given the nature of the above mentioned effects, specific quantifiers like "entropy" $\mathcal{H}_{S}$ and "complexity" $\mathcal{C}_{J S}$ could potentially reveal additional important aspects associated to the series-PDF that can not be discerned via the behavior of $\alpha$. We pass now to a discussion centered on the two just mentioned quantifiers.

For each one of the ten time-series generated for each pair $(k, A)$, the normalized Shannon entropy $\mathcal{H}_{S}$ and MPR statistical complexity measure $\mathcal{C}_{J S}$ were evaluated using i) the Bandt and Pompe PDF and ii) the procedure described in previous sections. Fig. 6 displays the pertinent values for series of length $N=10^{5}$ data and $D=6$. If $k \approx 0$ we see from these graphs that, for increasing values of the amplitude $A$, entropy and complexity values change (starting from the value corresponding to the pure logistic series, i.e., $A=0$ ) with a tendency to approach the values corresponding to pure noise, that is, $\left(\mathcal{H}_{S} \approx 1\right.$ and $\left.\mathcal{C}_{J S} \approx 0\right)$. As $k$ increases, the dependence on the noise-amplitude $A$ tends to become attenuated, and it almost disappears for $k \approx 2$. This is due to the effect of the "coloring" (correlations) of the noise, that grows with increasing values of $k$.

Using the values displayed in Fig. 6 we generate the associated causality entropy-complexity plane for each one of the relevant $k$ values and for different noise-amplitudes $A$. These planes are depicted in Fig. 7. We also display in these planes values pertaining to the pure-noise time series (with the same record length $N=10^{5}$ data) in the guise of open symbols. The continuous lines represent the values of minimum and maximum statistical complexity $\mathcal{C}_{\min }$ and $\mathcal{C}_{\max }$, respectively, evaluated for $D=6$.

Of course, for $A=0$ we obtain the purely deterministic value corresponding to the logistic time-series, localized approximately at a medium-hight entropic coordinate, near the highest possible complexity. Note that such is the typical behavior observed for deterministic systems [12]. For a purely uncorrelated stochastic process $(k=0)$ we have $\mathcal{H}_{S}=1$ and $\mathcal{C}_{J S}=0$. The correlated (colored) stochastic processes $(k \neq 0)$ yield points located at intermediate values between the curves $\mathcal{C}_{\text {min }}$ and $\mathcal{C}_{\text {max }}$, with decreasing values of entropy and increasing values of complexity as $k$ grows [12] (see Fig. 66).

Fig. 7 shows that the main effect of the additive noise $(A \neq 0)$ is to shift the point representative of zero noise $(A=0)$ towards increasing values of 
entropy $\mathcal{H}_{S}$ and decreasing values of complexity $\mathcal{C}_{J S}$. This shift defines a curve that is located in the vicinity of the maximum complexity $\mathcal{C}_{\max }-$ curve. Such behavior can be linked to the persistence of forbidden patterns because they, in turn, imply that the deterministic logistic component is still operative and influencing the TS-behavior.

We stress the fact that noise acts here as an additive perturbation to the logistic series. We may assert that some degree of robustness of our (causality) entropy-complexity plane against the effects of TS-noise contamination becomes evident. Moreover, a clear differentiation can be appreciated between the localization of the points corresponding to the time series with additive noise with those from a "pure-noise" series. We insist on the fact that such entropy-complexity plane's results are in agreement with the persistence of forbidden patterns from the deterministic component of the time-series.

Interestingly enough, if we plot these values (for all $A$ ) in a single graph, as in Fig. 8, we clearly appreciate the behavior described in the preceding paragraphs. Moreover, all the pertinent points yield a curve that closely approaches the maximum complexity one $\mathcal{C}_{\max }$. We can hypothesize that this curve is characteristic of the dynamical deterministic system, and could be used in order to determine the level of noise-contamination. Research in this direction is being currently undertaken.

\section{Conclusions}

We have looked carefully at the concept of forbidden/missing ordinal patterns. Why? Because it has been recently used as a tool for the discrimination between deterministic and stochastic behavior in observational time series [14,15, 16, 20,21,19]. Given the importance of the subject, that in the light of these previous efforts becomes evident, in the present work we have extended the analysis of missing ordinal patterns [17,18] and linked it to the causality entropy-complexity plane [12]. Our considerations were made with regards to deterministic finite time series contaminated (as a perturbation) with additive noises of different degree of correlation.

We were able to show that, by comparing the decay rate of missing ordinal patterns both in a noisy time series and in a pure-noise (with some degree of correlation) one, insights pertaining to the deterministic component of the original time series can be gained.

This seemingly worthy goal was achieved, without the need of additional numerical work such as the one needed for evaluating the decay rate of missing ordinal patterns. It just suffices to look carefully at the planar locations of 
the pertinent points, for each time series. Moreover, we have encountered a characteristic behavior that can be associated to variations in the amount of noise-contamination which, in turn, facilitates the identification of the timeseries' deterministic component.

\section{Acknowledgments}

This research has been partially supported by a scholarship from The University of Newcastle awarded to Laura C. Carpi. Osvaldo A. Rosso gratefully acknowledges support from CAPES, PVE fellowship, Brazil. Martín Gómez Ravetti acknowledges support from FAPEMIG and CNPq, Brazil.

\section{References}

[1] H. Kantz, T. Scheiber, Nonlinear Time Series Analysis. Cambridge University Press, Cambridge, UK, 2002.

[2] H. D. I. Abarbanel, Analysis of Observed Chaotic Data. Springer-Verlag, New York, USA, 1996.

[3] A. N. Kolmogorov, A new metric invariant for transitive dynamical systems and automorphisms in lebesgue sapces. Dokl. Akad. Nauk., USSR , 119 (1959) 861 864.

[4] Y. G. Sinai, On the concept of entropy for a dynamical system. Dokl. Akad. Nauk., USSR , 124 (1959) $768-771$.

[5] A. R. Osborne, A. Provenzale, Finite correlation dimension for stochastic systems with power-law spectra. Physica D 35 (1989) 357-381.

[6] P. Grassberger, I. Procaccia, Measuring the strangness of strange attractors. Physica D 9 (1983) 189-208.

[7] G. Sugihara, R. M. May, Nonlinear forecasting as a way of distinguishing chaos from measurement error in time series. Nature 344 (1983) 734-741.

[8] D. T. Kaplan, L. Glass, Direct test for determinism in a time series. Phys. Rev. Lett. 68 (1992) 427-430.

[9] D. T. Kaplan, L. Glass, Coarse-grained embeddings of time series: random walks, Gaussian random processes, and deterministic chaos. Physica D 64 (1993) 431454 .

[10] H. Kantz, E. Olbrich, Coarse grained dynamical entropies: Investigation of highentropic dynamical systems. Physica A 280 (2000) 34-48. 
[11] M. Cencini, M. Falcioni, E. Olbrich, H. Kantz, A. Vulpiani, Chaos or noise: Difficulties of a distinction. Physica A 280 (2000) 34-48.

[12] O. A. Rosso, H. A Larrondo, M. T. Martín, A. Plastino, M. A. Fuentes, Distinguishing noise from chaos. Phys. Rev. Lett. 99 (2007) 154102.

[13] C. Bandt, B. Pompe, Permutation entropy: a natural complexity measure for time series. Phys. Rev. Lett. 88 (2002) 174102.

[14] J. M. Amigó, L. Kocarev, J. Szczepanski, Order patterns and chaos Phys. Lett. A 355 (2006) 27-31.

[15] J. M. Amigó, S. Zambrano, M. A. F. Sanjuán, True and false forbidden patterns in deterministic and random dynamics. Europhys. Lett. 79 (2007) 50001.

[16] J. M. Amigó, S. Zambrano and M. A. F. Sanjuán, Combinatorial detection of determinism in noisy time series. Europhys. Lett. 83 (2008) 60005.

[17] J. M. Amigó, Permutation complexity in dynamical systems. Springer-Verlag, Berlin, Germany, 2010.

[18] L. C. Carpi, P. M. Saco, O. A. Rosso, Missing Ordinal Patterns in Correlated Noises. Physica A 389 (2010) 2020-2029.

[19] M. Zanin, Forbidden patterns in financial time series. Chaos 18 (2008) 013119.

[20] L. Zunino, M. Zanin, B. M. Tabak, D. Pérez, O. A. Rosso, Forbidden patterns, permutation entropy and stock market inefficiency. Physica A 388 (2009) 2854 2864 .

[21] G. Ouyang, X. Li, C. Dang and D. A. Richards, Deterministic dynamics of neural activity during absence seizures in rats. Phys. Rev. E 79 (2009) 041146.

[22] C. Shannon, W. Weaver, The Mathematical Theory of Communication University of Illinois Press, Champaign, IL, 1949.

[23] D. P. Feldman, J. P. Crutchfield, Measures of statistical complexity: Why?. Phys. Lett. A 238 (1998) $244-252$.

[24] D. P. Feldman, C. S. McTague, J. P. Crutchfield, The organization of intrinsic computation: Complexity-entropy diagrams and the diversity of natural information processing. Chaos 18 (2008) 043106.

[25] P. W. Lamberti, M. T. Martín, A. Plastino, and O. A. Rosso, Intensive entropic nontriviality measure. Physica A 334 (2004) 119 - 131.

[26] R. López-Ruiz, H. L. Mancini, X. Calbet, A statistical measure of complexity. Phys. Lett. A 209 (1995) $321-326$.

[27] I. Grosse, P. Bernaola-Galván, P. Carpena, R. Román-Roldán, J. Oliver, H. E. Stanley, Analysis of symbolic sequences using the Jensen-Shannon divergence. Phys. Rev. E 65 (2002) 041905.

[28] A. R. Plastino, and A. Plastino, Symmetries of the Fokker-Plank equation and Fisher-Frieden arrow of time. Phys. Rev. E 54 (1996) 4423 - 4326. 
[29] M. T. Martín, A. Plastino, O. A. Rosso, Generalized statistical complexity measures: geometrical and analytical properties. Physica A 369, 439 (2006) 439 462 .

[30] O. A. Rosso, C. Masoller, Detecting and quantifying stochastic and coherence resonances via Information Theory complexity measurements. Phys. Rev. E 79 (2009) 040106(R)

[31] O. A. Rosso, L. de Micco, H. Larrondo, M. T. Martín, A. Plastino, Generalized statistical complexity measure. Int. J. Bif. and Chaos 20 (2010) 775-785.

[32] L. Zunino, M. Zanin, B. M. Tabak, D. G. Pérez, O. A. Rosso, Complexityentropy causality plane: A useful approach to quantify the stock market inefficiency. Physica A 389 (2010) 1891-1901.

[33] L. Zunino, B. M. Tabak, F. Serinaldi, M. Zanin, D. G. Pérez, O. A. Rosso, Commodity predictability analysis with a permutation information theory approach.

[34] O. A. Rosso, H. Craig, P. Moscato, Shakespeare and other English renaissance authors as characterized by Information Theory complexity quantifiers. Physica A 388 (2009) $916-926$

[35] L. De Micco, C. M. González, H. A. Larrondo, M. T. Martín, A. Plastino, O. A. Rosso, Randomizing nonlinear maps via symbolic dynamics. Physica A 87 (2008) $3373-3383$.

[36] K. Mischaikow, M. Mrozek, J. Reiss, A. Szymczak, Construction of symbolic dynamics from experimental time series. Phys. Rev. Lett. 82 (1999) 1114 - 1147.

[37] G. E. Powell, I. C. Percival, A spectral entropy method for distinguishing regular and irregular motion of hamiltonian systems. J. Phys. A: Math. Gen. 12 (1979) $2053-2071$.

[38] O. A. Rosso, S. Blanco, J. Jordanova, V. Kolev, A. Figliola, M. Schürmann, E. Başar, Wavelet entropy: a new tool for analysis of short duration brain electrical signals. J. Neurosc. Meth. 105 (2001) 65-75.

[39] J. M. Finn, J. D. Goettee, Z. Toroczkai, M. Anghel, B. P. Wood, Estimation of entropies and dimensions by nonlinear symbolic time series analysis. Chaos 13, 444 (2003) $444-457$.

[40] E. M. Bollt, T. Stanford, Y. C. Lai, K. Życzkowski, Validity of ThresholdCrossing Analysis of Symbolic Dynamics from Chaotic Time Series. Phys. Rev. Lett. 85 (2000) $3524-3527$.

[41] C. S. Daw, C. E. A. Finney, E. R. Tracy, A review of symbolic analysis of experimental data. Rev. Sci. Instrum. 74 (2003) 915 - 931.

[42] H. Wold, A Study in the Analysis of Stationary Time Series. Almqvist and Wiksell, Upsala, Sweden, 1938. 
[43] J. Kurths, H. Herzel, An attractor in a solar time series. Physica D 25 (1987) $165-172$.

[44] S. Cambanis, C. D. Hardin, A. Weron, Innovations and Wold decompositions of stable sequences. Probab. Theory Relat. Fields 79 (1988) $1-27$.

[45] K. Keller, M. Sinn, Ordinal analysis of time series, Physica A 356 (2005) 114120.

[46] P. M. Saco, L. C. Carpi, A. Figliola, E. Serrano, O. A. Rosso, Entropy analysis of the dynamics of El Niño/Southern Oscillation during the Holocene. Physica A 389 (2010) $5022-5027$.

[47] In example, consider the two pure stochastic time series generated by the following recursive relation: (a) $Y(t+1)=Y(t) \cdot X(t), X$ is a random variable, $X \sim \mathrm{U}[a, b]$, with $a, b \in \mathbb{R}^{+}$and $b>a>1$. (b) $Y(t+1)=(-1)^{t} \cdot X(t), X$ is a random variable, $X \sim \mathrm{U}[a, b]$, with $a, b \in \mathbb{R}^{+}$. Both random time series have forbidden patterns.

[48] C. Bandt, F. Shisha. Order patterns in time series, J. Time Ser. Anal. 28 (2007) 646-665.

[49] J. C. Sprott, Chaos and Time Series Analysis, Oxford University Press, Oxford, 2004.

[50] M. Matsumoto, T. Nishimura, Mersenne twister: a 623-dimensionally uniform pseudo-random number gererator. ACM Transactions on Modeling and Computer Simulation 8 (1998) $3-30$. 

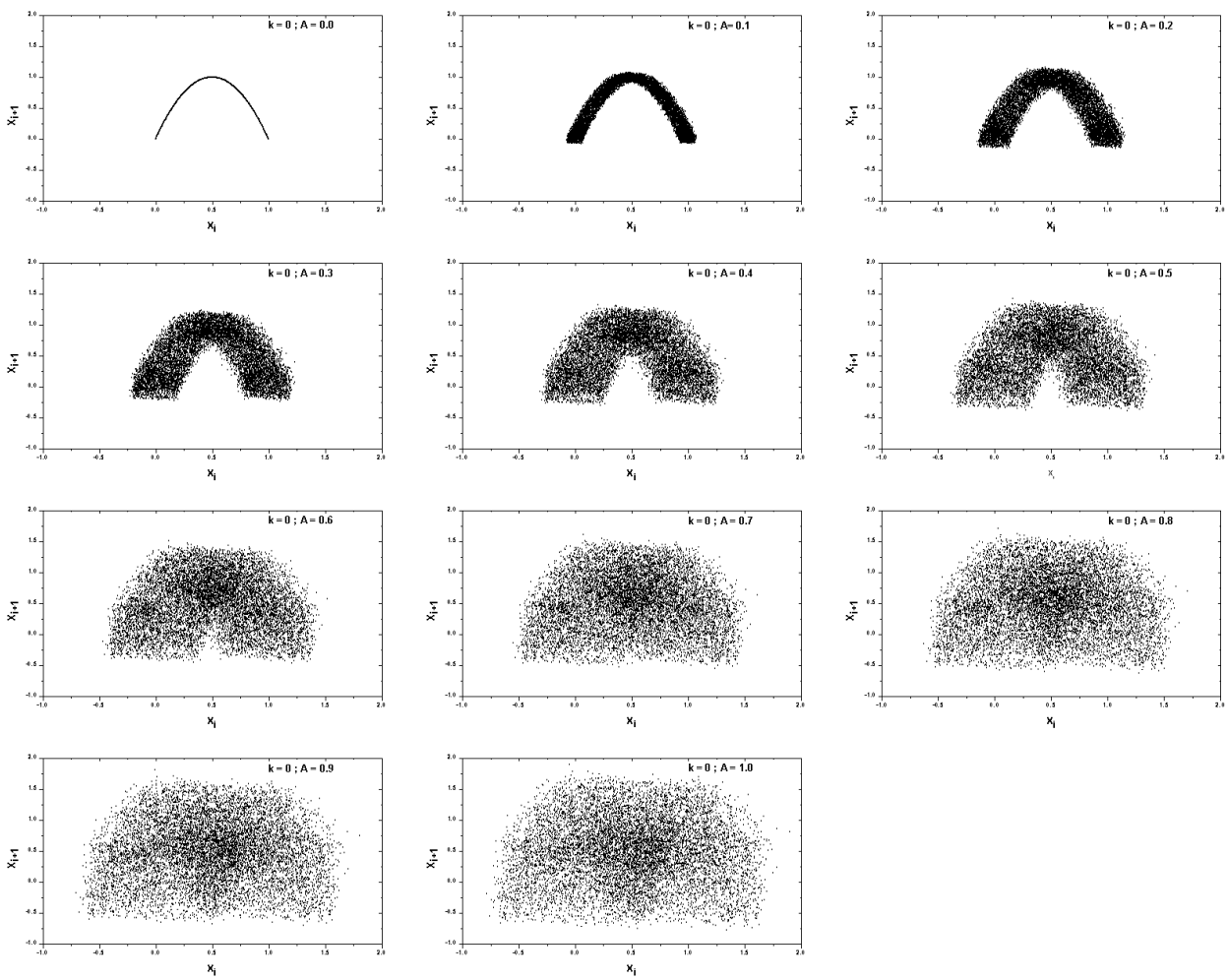

Fig. 1. First return map corresponding to a typical signal from a noise-contaminated logistic map $(r=4)$. The additive noise has a power spectrum of the $f^{-k}$-type $\left(N=10^{4}\right.$ data). We consider the case $k=0$ (white noise) with noise amplitude $0 \leq A \leq 1$. 

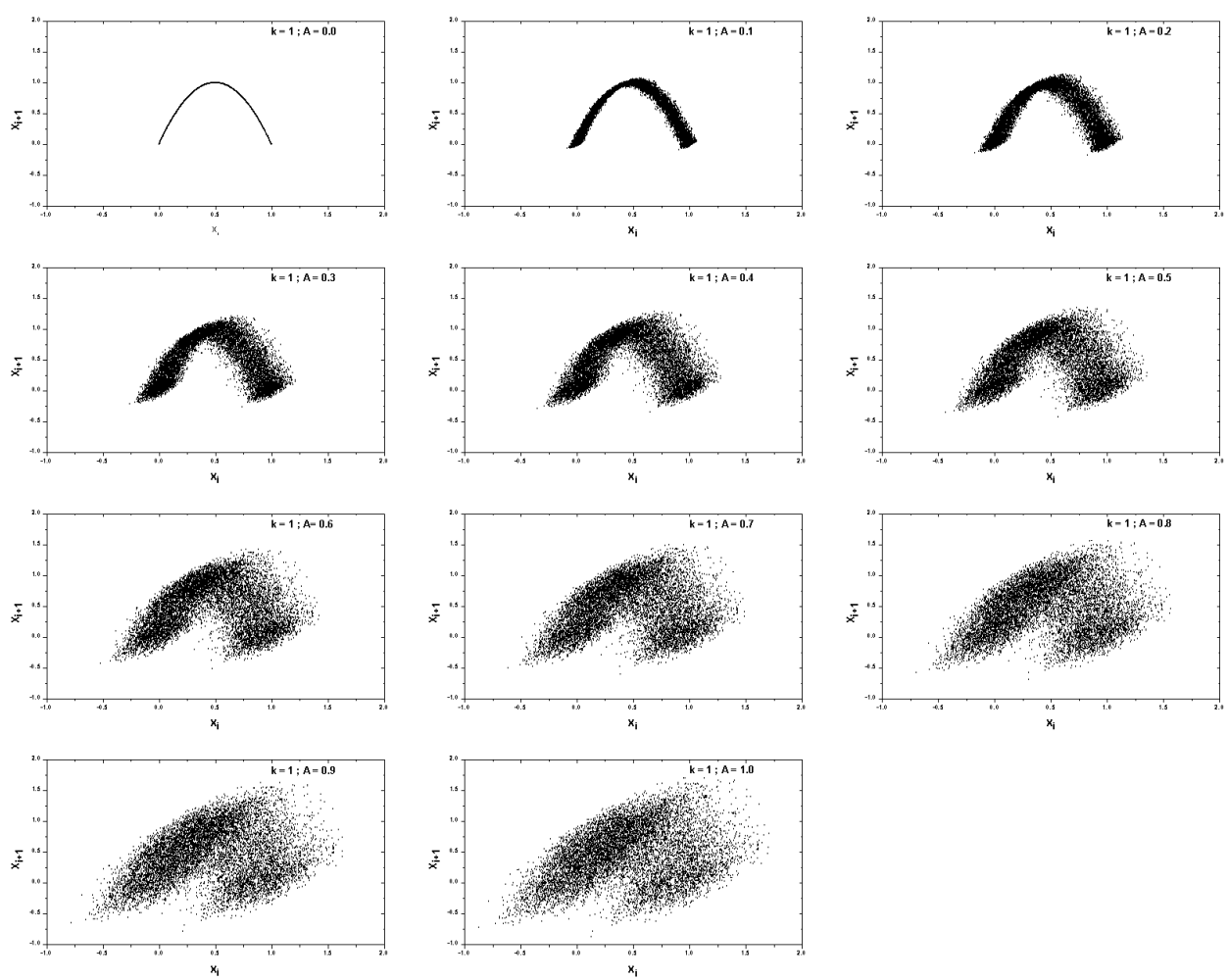

Fig. 2. Same as in Fig. 1 but for $k=1$. 

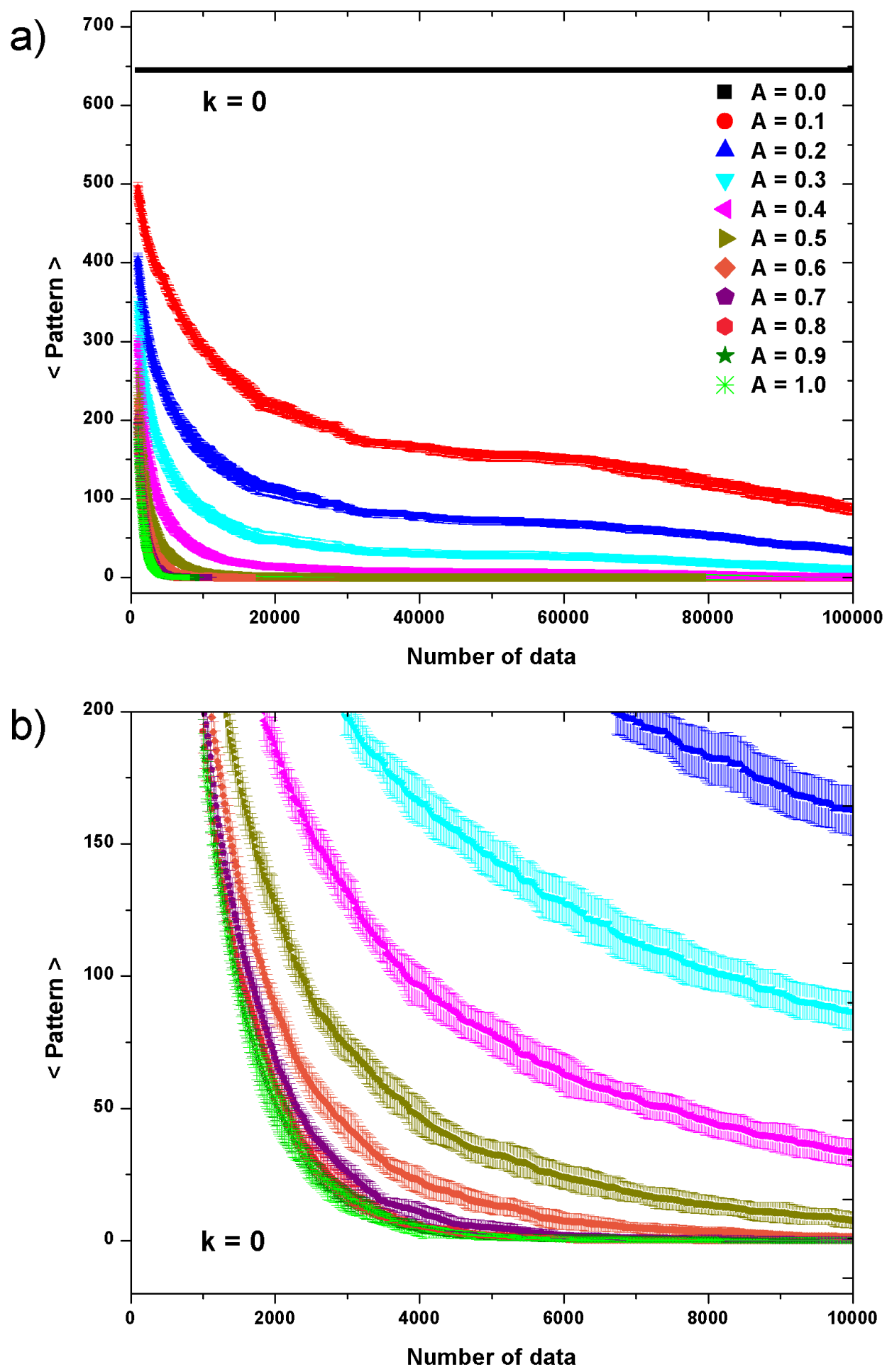

Fig. 3. a) Average over ten time series of the missing patterns number $<\mathcal{M}(N, D)>($ mean $\pm S D)$, as a function of the time series length $10^{3} \leq N \leq 10^{5}$, for the case $k=0$ (withe noise) and $D=6$. Note that for the case $A=0$ we have a purely logistic time series and the associated number of missing patterns $<$ Pattern $>=645$ is independent of the time series length. $b$ ) Details over $10^{3} \leq N \leq 10^{4}$. 

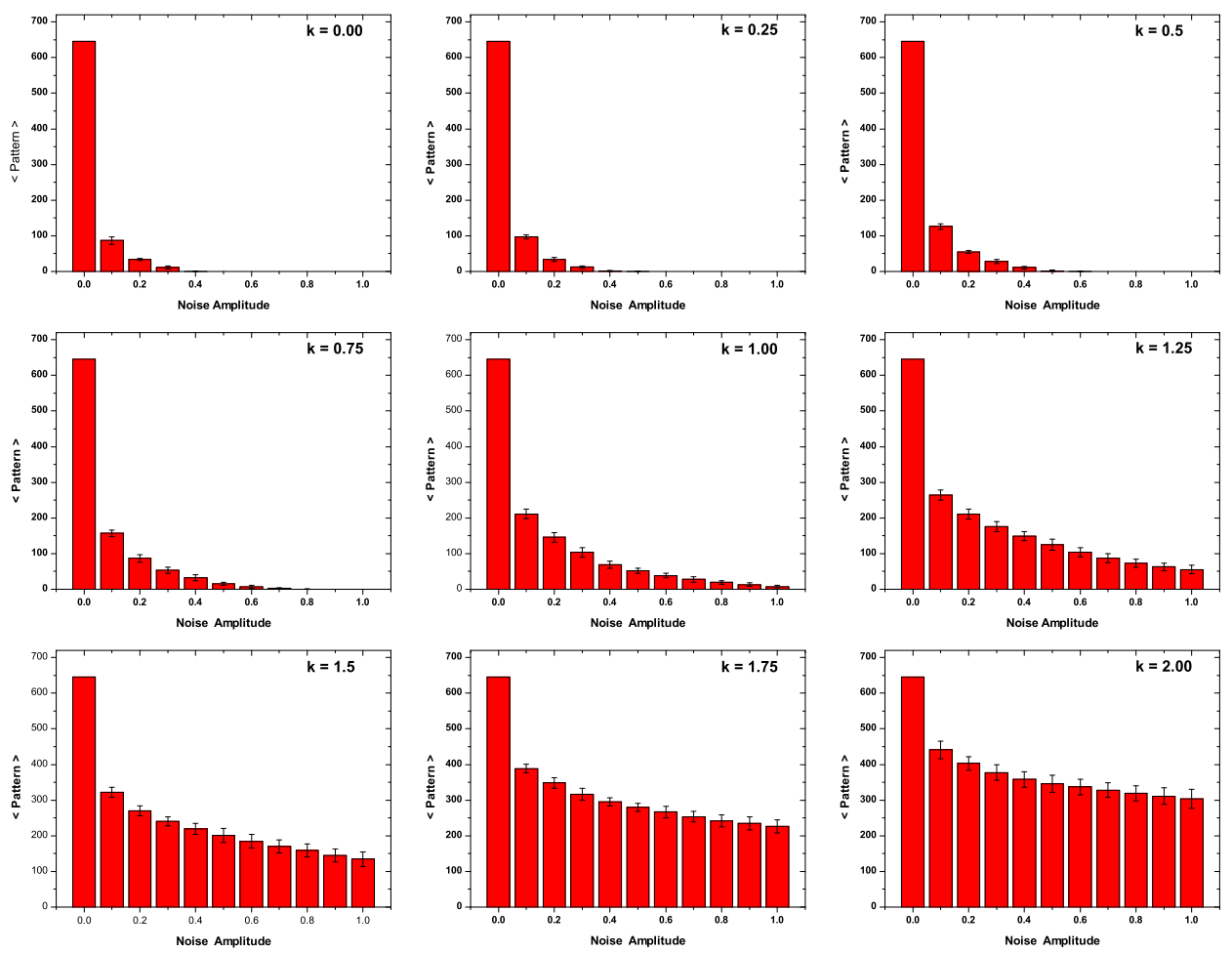

Fig. 4. Average values corresponding to the number of missing patterns $<\mathcal{M}(N, D)>($ mean $\pm S D)$ for times series of $N=10^{5}$ data and $D=6$, as a function of the noise amplitude $A$ and for several $k$ values.

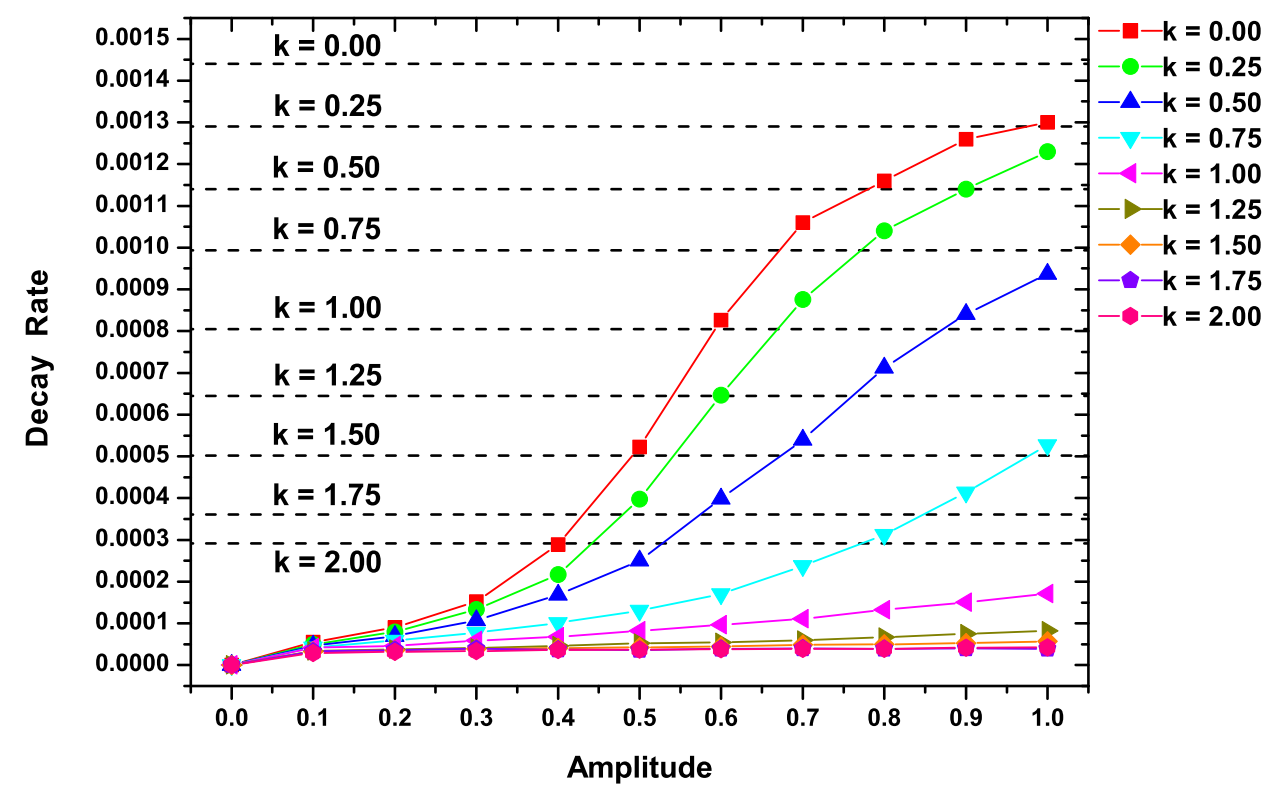

Fig. 5. Decay rate $\alpha$ for the missing patterns of the noise-contaminated logistic time series, for different values of $k$ as a function of the additive noise component $A$. Horizontal lines correspond to the pure noise-value with $f^{-k}$ power spectrum. The times series' length is $10^{3} \leq N \leq 10^{5}$ data and the pattern length used is $D=6$. 
a)

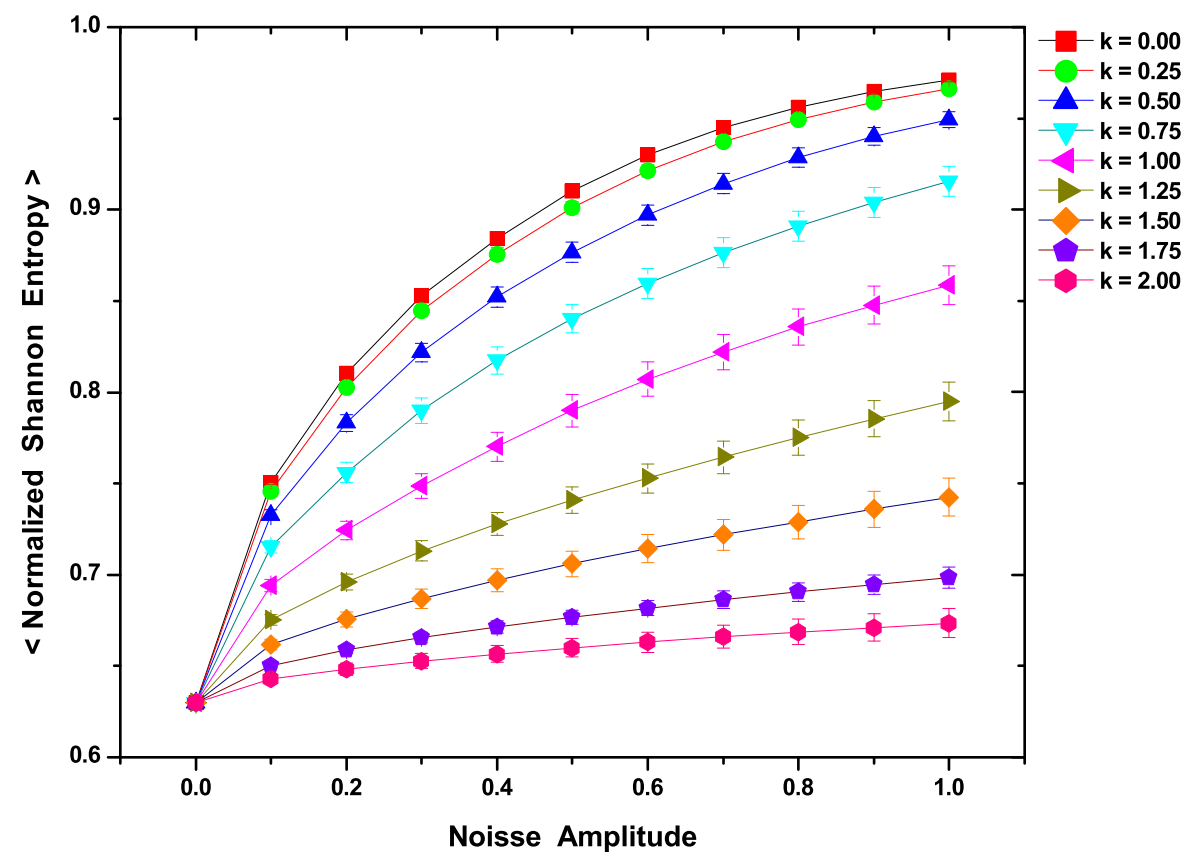

b)

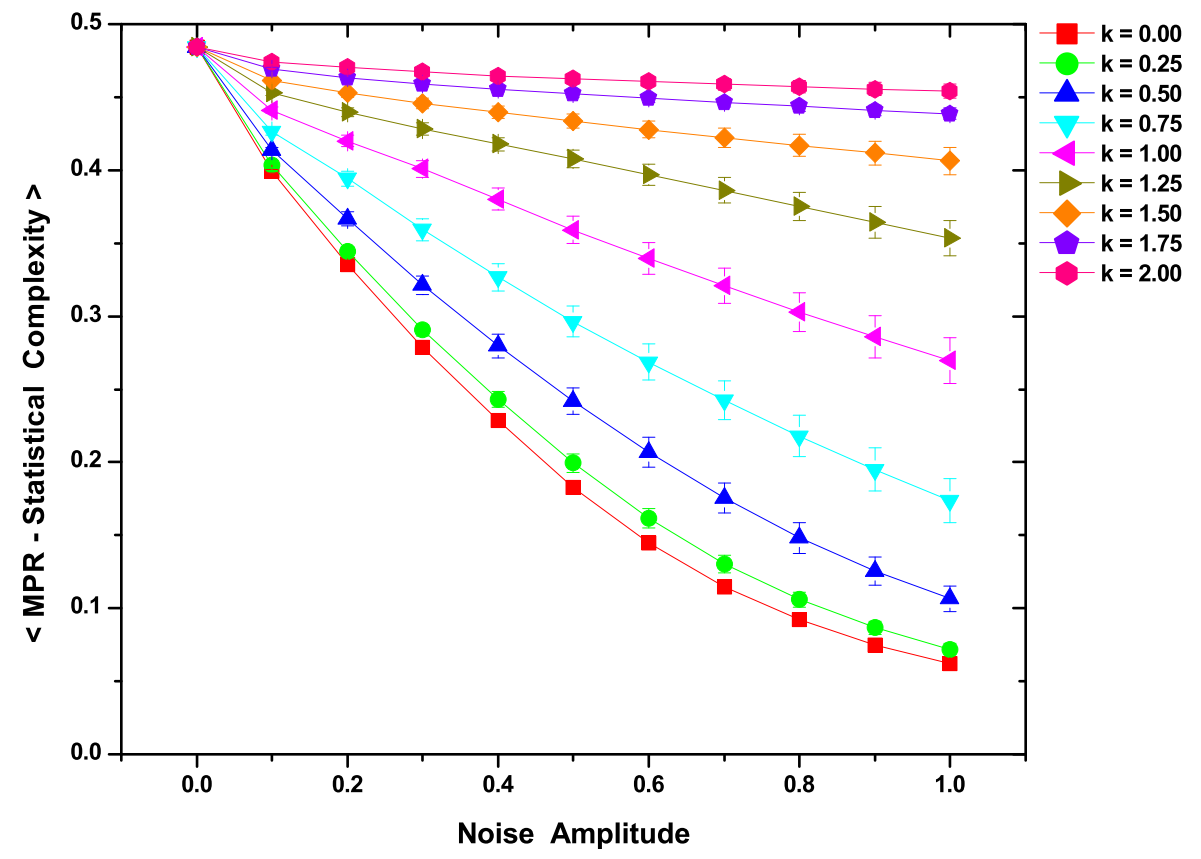

Fig. 6. Average values (mean $\pm S D$ ) corresponding to $(a)$ the normalized Shannon entropy $\mathcal{H}_{S}$ and $(b)$ the statistical complexity $\mathcal{C}_{J S}$, for times series of $N=10^{5}$ data and $D=6$, as a function of the noise amplitude $A$ and for several $k$ values. 

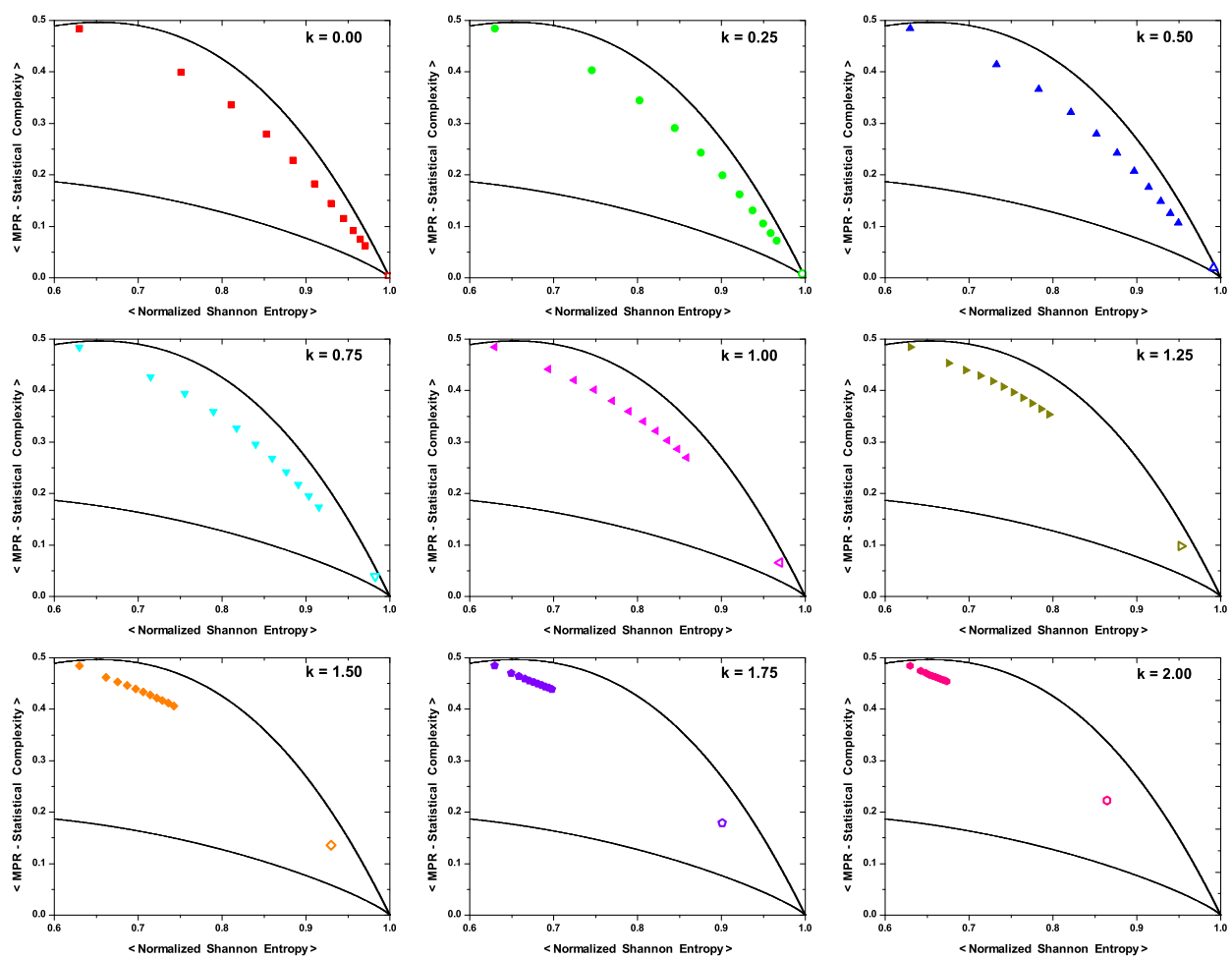

Fig. 7. The causality entropy-complexity plane associated to Fig. 6 for different values of both $k$ and $A$ (times series length $N=10^{5}$ data and $D=6$ ). The corresponding values for a purely noisy series $\left(N=10^{5}\right.$ data and $\left.D=6\right)$ are also displayed in the guise of open symbols. The continuous lines represent the values of minimum and maximum statistical complexity $\mathcal{C}_{\text {min }}$ and $\mathcal{C}_{\text {max }}$, evaluated for the case of pattern length $D=6$. In each graph, the first point at the west has $A=0$ (to the left) and the last at the east $A=1$ (to the right). 
a)

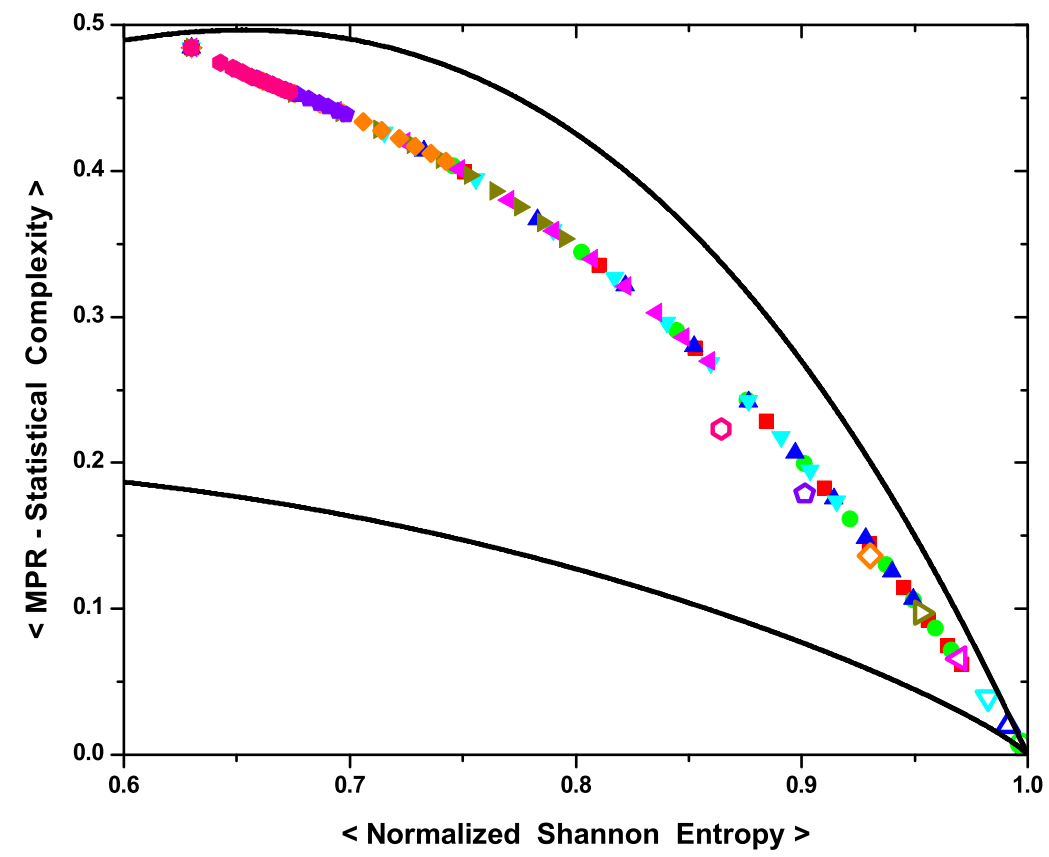

b)

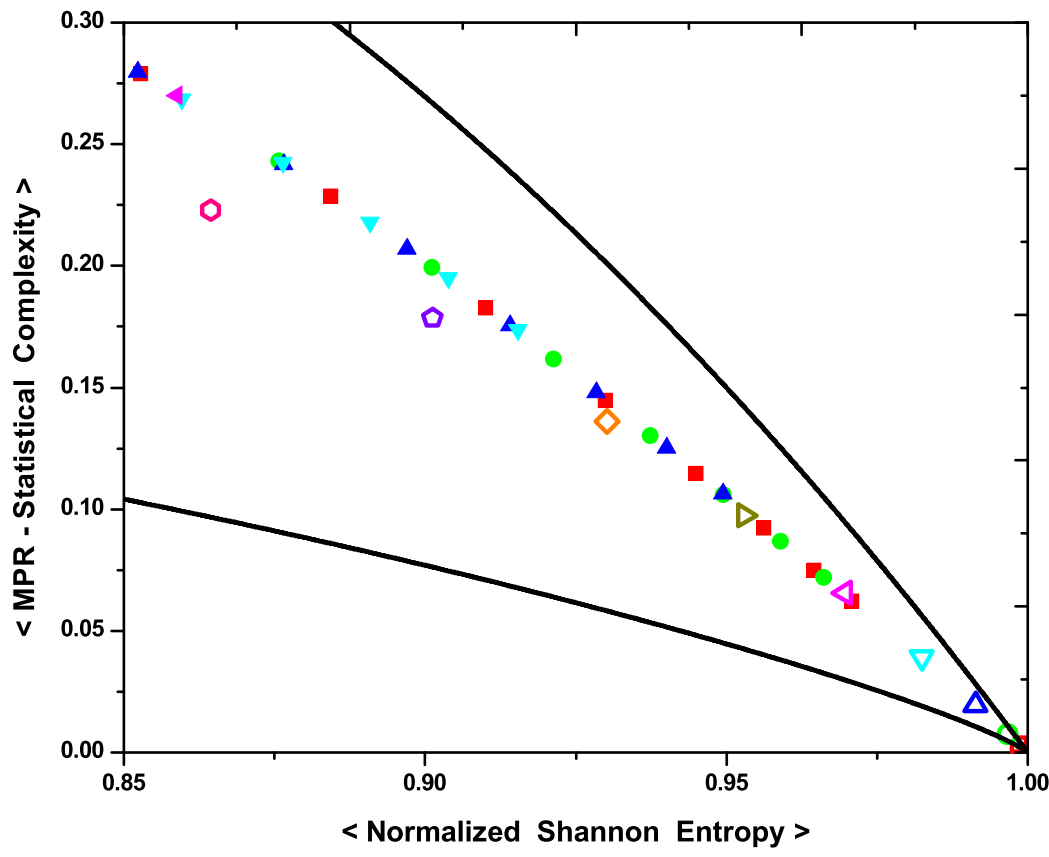

$k=0.00$

$k=0.25$

$\Delta \mathrm{k}=\mathbf{0 . 5 0}$

$\mathbf{k}=\mathbf{0 . 7 5}$

$k=1.00$

$k=1.25$

$k=1.50$

$k=1.75$

$k=2.00$

Shannon Entropy >

Fig. 8. (a) The causality entropy-complexity plane for all values displayed in (Fig. 6) corresponding to different of both $k$ and $A$ (times series length $N=10^{5}$ data and $D=6)$. Values for the case of strictly noisy time series $\left(N=10^{5}\right.$ data and $D=6$ ) are also shown as open symbols. The continuous lines represent the values of minimum and maximum statistical complexity $\mathcal{C}_{\min }$ and $\mathcal{C}_{\text {max }}$ evaluated for the case of pattern length $D=6$. (b) Amplification. 